\title{
Longitudinal Assessment of Critical Thinking Skills Across a Dental Curriculum
}

\begin{abstract}
Allison C. Everett, Olivia S. Anderson, Mary C. Wright, Margherita Fontana
Abstract: Critical thinking and problem-solving are two of the most frequently desired goals for dental student learning, but greater understanding is needed of how students' critical thinking skills develop from the beginning to the end of the curriculum. The aim of this study was to use students' performance on critical thinking assessments at five time points from the first to the fourth years to longitudinally assess their critical thinking development across the curriculum. In 2014-16, the performance of students in two successive graduating classes at one U.S. dental school was collected from five assignments designed to, among other things, assess critical thinking. Those assignments ranged from a case study in the Cariology I course in fall of the first year to an OSCE completed before graduation. Students were divided into four quartiles from lowest- to highest-achieving based on the first (baseline) assessment, and those quartiles were tracked to determine each student's progress over the four years. The results showed that, for both cohorts, students who ranked in the top quartile remained in the top of the class for all subsequent assessments. Also in both cohorts, the students who had the lowest scores on the initial assessment of critical thinking skills showed statistically significant improvements over time, increasing their scores by $9.4 \%$ (cohort $1, \mathrm{p}<0.001$ ) and $4.6 \%$ (cohort $2, p=0.003)$ in each consecutive assessment. These results suggest that implementing critical thinking assessments early in the curriculum can assist in predicting students' future performance on critical thinking assessments. Furthermore, a dental curriculum intentionally designed to promote critical thinking capacities can help students improve these skills over time, whatever their level at the beginning.
\end{abstract}

Allison C. Everett, DDS, is Clinical Assistant Professor, Loyola University Medical Center; Olivia S. Anderson, PhD, RD, is Clinical Assistant Professor, Department of Nutritional Sciences, School of Public Health, University of Michigan; Mary C. Wright, $\mathrm{PhD}$, is Director, Sheridan Center for Teaching and Learning, Brown University; and Margherita Fontana, DDS, PhD, is Professor, School of Dentistry, University of Michigan. Direct correspondence to Dr. Margherita Fontana, Department of Cariology, Restorative Sciences, and Endodontics, School of Dentistry, University of Michigan, 1011 N. University, 2393, Ann Arbor, MI 48109-1078; 734-647-1225; mfontan@umich.edu.

Keywords: dental education, critical thinking, assessment, curriculum

Submitted for publication 11/15/17; accepted 2/10/18

doi: 10.21815/JDE.018.088

$\mathrm{H}$ elping students develop critical thinking skills is a key component of contemporary education. In a survey of U.S. employers published in 2013, $82 \%$ of respondents agreed that more curricular emphasis should be placed on critical thinking and analytical reasoning. ${ }^{1}$ In dental education, the Commission on Dental Accreditation (CODA) now requires that predoctoral dental programs emphasize critical thinking and problemsolving in educating future dentists. ${ }^{2}$

Chambers defined critical thinking as "being able to give reasons for what one says and does" (p. 65). ${ }^{3}$ The American Dental Education Association Commission on Change and Innovation in Dental Education (ADEA CCI) notes that critical thinking "is also regarded as intellectually engaged, skillful, and responsible thinking that facilitates good judgment because it requires the application of assumptions, knowledge, competence, and the ability to challenge one's own thinking. ${ }^{\prime 4}$ In 2015, the University of Michigan School of Dentistry adopted the following definition to guide instruction and assessment of student performance: "The ability to think critically involves: 1) an attitude of being included to consider in a thoughtful way the problems and subjects that come within the range of one's experiences, 2) knowledge of the methods of logical inquiry and reasoning, and 3) skill in applying those methods." Critical thinking requires more than recitation of learned material because it involves reflection as well as application of pertinent knowledge and recent evidence to make appropriate judgments in clinical scenarios.

To develop critical thinking skills, the ADEA CCI suggests that dental schools should provide opportunities for students to engage in problem-solving, analysis of case-based scenarios, debates, role-play, argument mapping, thinking aloud, reflection, and simulation, among other activities. ${ }^{4}$ Overall, dental curricula should incorporate lessons and activities that encourage critical thinking, reflective practice, and their application to clinical decision making. ${ }^{3}$ 
Also, since critical thinking is a skill developed over time, understanding how that process develops would be useful for dental educators in planning lessons and activities across the curriculum.

The aim of this study was to use students' performance on critical thinking assessments at five time points from the first to the fourth years to longitudinally assess their critical thinking development across the curriculum. In this longitudinal study, we sought to answer questions like these: Did students improve their critical thinking capacities over time in a curriculum that offered deliberate opportunities for practice? Did higher-performing students in the first year maintain their high achievement? Did lower-performing students in the first year improve their achievement? Our long-term goal is to offer a model for dental schools to measure students' critical thinking gains.

\section{Methods}

The Institutional Review Board at the University of Michigan approved this study (IRB \# HUM00018003). The participants whose data were analyzed in the study were two consecutive University of Michigan School of Dentistry cohorts of students (graduating classes of 2014 and 2015), identified as cohort 1 and cohort 2. All students in each cohort (Class of $2014 \mathrm{~N}=102$ and Class of 2015 $\mathrm{N}=107$ ) were informed of the study and given the opportunity to opt out. Data were collected in 2014-16.

The first step was to select student assessments that required critical thinking capacities across the four years of the DDS curriculum. To determine whether an assessment tested critical thinking, we used four criteria representing the main components of critical thinking defined in authoritative sources. ${ }^{3-8}$ The selected assessments had to ask students to 1) analyze and evaluate information; 2) apply assumptions, knowledge, and competence; 3) pursue the best possible option for a situation; and 4) prompt students to give reasons for decisions. Thus, they had to measure higher order thinking skills (application, analysis, synthesis, and evaluation), according to Bloom's taxonomy, in a written or multiple-choice format. ${ }^{9}$

Course directors were asked to help identify assessments in their courses that fit the criteria. We evaluated the assessments proposed by the course directors and discussed them with the Office of Aca- demic Affairs to identify any missing information. Multiple courses that incorporated critical thinking were identified. In the first year, the Cariology I and II courses used final examinations and case studies that required and evaluated critical thinking. In the second year, an objective structured clinical exam (OSCE) and treatment planning course provided opportunities for students to further develop critical thinking skills. In the third and fourth years, students were tested on their ability to evaluate, develop, and justify a treatment plan for patients, as well as complete required restorative, prosthodontic, and periodontic test cases in the comprehensive care clinics. Also, in the third year, students develop a treatment plan and justify their rationale with evidence for a geriatric patient, and in the fourth year, students must pass an OSCE. Some of these opportunities for critical thinking were subjectively graded or did not have a standarized score and were thus not eligible for our study. Every effort was made to identify at least one assessment (or portion of an assessment) per year for which an individual numerical grade per student could be extracted.

Five assessments were selected from across the four years. The assessments incorporated high-level cognitive multiple-choice questions or short answers that included creating and justifying a treatment plan based on a patient scenario. The first (baseline) assessment was a case study in the Cariology I course in fall of the first year. The case study was students' first patient case scenario in this course in which they had to analyze images and/or radiographs and provide information to justify the rationale for the patient's caries risk, contributing factors, and selection and justification of an appropriate evidence-based caries management plan. Each case was scored using a rubric that assessed students' ability, rationale, and evidence to associate caries detection, diagnosis, and risk with their management plan.

The rubric for the baseline assessment was organized into three categories: caries experience, caries risk assessment, and caries management (Table 1). For caries experience, students were required to identify and stage the severity of carious lesions, explain how they could determine if those caries lesions were active or arrested, and justify their decisions for the case. For caries risk assessment, students had to identify contributing factors to the patient's caries experience (e.g., caries experience, diet, saliva, plaque, protective factors, compliance), decide on the patient's caries risk and an appropriate 
Table 1. Grading rubric used in cariology case in the baseline assessment

\begin{tabular}{|c|c|}
\hline Topic & Description of Activity \\
\hline $\begin{array}{l}\text { Caries } \\
\text { experience }\end{array}$ & $\begin{array}{l}\text { Examine images provided and assess the occlusal } \\
\text { surface of tooth } \# X X \text { and XX. Determine if surfaces } \\
\text { are sound or if there is any associated pathology. } \\
\text { Be sure to use correct terminology, both for caries } \\
\text { lesions and other hard tissue defects. If describing } \\
\text { caries lesions, refer to both severity and activity } \\
\text { (and provide a rationale for your diagnostic call; } \\
\text { e.g., in this surface I believe there is a lesion of } \\
\text { this severity, active or arrested, because ...) }\end{array}$ \\
\hline
\end{tabular}

Caries risk assessment

Complete a caries risk assessment for the patient in this case (you may use the UoM form or others as guidance, but do not submit these forms. Risk determination cannot be based on "because the form told $\mathrm{me}^{\prime \prime}$ ):

- Identify the patient's caries risk factors.

- Define and justify the patient's caries risk status (low, moderate, or high)

- Provide a recommended recall interval for caries management (3 months, 6 months, annual).
Caries Develop a detailed caries management plan for management this patient (you may use the UoM form as guidance, but do not include these forms):

- It is expected that every existing risk factor will be linked to a management recommendation; if not, there will be a rationale why not. Provide a rationale for each element of the management plan. For products to be used, provide concentration, active ingredient, and how and when the patient is to use it.

- Provide evidence-based resources to support each recommendation in your caries management plan. Use and cite the highest quality, strong evidence resources you can find (2 for each specific recommendation).

- Rank management plan elements based on BOTH the sequence of treatment and the strength of the evidence (the rationale for the ranking must be clear).
Scoring Rubric

2 points ( 1 point per tooth: 0.5 for correct severity score, 0.5 for correct activity score, including correct rationale). However, if rationale/explanation is correct but score/ activity provided is wrong: 0.5 per tooth.

Total: 8 points

Caries experience, diet, saliva, plaque, protective factors, compliance (4 points):

- If missing risk factors (2 or more) (-2)

- If no mention of future orthodontic treatment (-2) (note: patient in case will start ortho treatment next week)

Caries risk (3 points):

- Risk level is not supported by rationale student provides (-1)

- If classified patient as high risk (-2) (note: based on case, patient is currently low risk; once ortho treatment starts, risk will be raised to moderate)

Recall interval (1 point):

- No recall mentioned, or inappropriate recall based on risk level (-1)

Total: 5 points

- If no in-office fluoride for moderate/high risk (-1)

- If ranking of treatment options based on existing evidence and rationale is incorrect (-1)

- If there are management solutions provided for risk factors not identified in previous sections $(-1)$

- If patient is classified as low risk, yet a caries management plan to change existing risk factors is included (-0.5 for every treatment provided besides general diet education)

- If moderate/high risk and no fluoride treatment modality is included (-1.5)

- If rationale for treatment is clearly incorrect $(-0.5)$

Note: The case included a description of the patient, clinical images, and radiographs.

recall, and justify the rationale and evidence for their answers. In the caries management portion, students had to provide appropriate management strategies based on the patient's current contributing factors and caries management and to justify the rationale and evidence for their answers. The scoring was designed to assess the student's ability to relate all three sections.

The second assessment was the final examination in the Cariology I course. This exam had been independently verified to incorporate high cognitive- level multiple-choice questions (based on patient scenarios and application of learned information). ${ }^{9}$ Cariology courses at the University of Michigan School of Dentistry prepare students to be able to perform dental caries detection, diagnosis, risk assessment, prevention, and management during clinical care throughout their dental education and when they become practitioners. ${ }^{10}$

The third, fourth, and fifth assessments took place in the second, third, and fourth years of the curriculum. The third assessment was the final 
examination in the second-year Treatment Planning course. That exam included two questions that required formulating and justifying a treatment plan for a patient scenario. The fourth assessment was a treatment plan for a patient in the third-year Geriatric Dentistry course. Students are required to find a geriatric patient in their patient family, analyze factors that could contribute to the patient's dental diseases, and develop and justify a treatment plan for the patient. The final assessment was an OSCE, completed prior to graduation, that includes high cognitive-level multiple-choice questions for each clinical discipline. The OSCE has been recommended as a testing method to identify mastery of critical thinking and problem-solving throughout a curriculum. ${ }^{11,12}$

After the courses, assessments, and students were identified, course directors submitted their students' grades on the specified assessments to one of the investigators (MF) through a secure server. Each student in each class cohort was assigned a random number. The assessments were blinded before sharing with the other investigators for analyses. Students were ranked in each cohort based on their Cariology I case study score (first assessment) and divided into quartiles (quartile 1 at the bottom, then quartiles 2 and 3 , with quartile 4 representing the top or best scores). The gathered data for both cohorts were analyzed using SPSS Version 22 (IBM Corp., Armonk, NY, USA). Linear regressions were completed for the entire cohort and with each cohort split into quartiles. The threshold for statistical significance was $\mathrm{p}<0.05$.

\section{Results}

Only students for which data were available for all five assessments were included. One student in cohort 1 declined to participate, so that student's data were not included in any of the analyses. Data from 101 students in cohort 1 and 107 students in cohort 2 were therefore analyzed.

The mean percentage and standard deviation on each assessment by cohort are shown in Table 2. Data are presented as percentage of correct response rather than actual scores, as the grading scales used in the assessments were not uniform (e.g., 1-100 vs. 1-5).

The thresholds used to define the four quartiles for each cohort based on the baseline assessment (Cariology I patient case critical thinking assessment) were as follows. For quartile 1, the range was $0-70 \%$ for cohort 1 and $0-76.67 \%$ for cohort 2 . For quartile 2, the range was $70.01-76.67 \%$ for cohort 1 and $76.68-80 \%$ for cohort 2 . For quartile 3 , the range was $76.68-86.67 \%$ for cohort 1 and $80.01-86.67 \%$ for cohort 2. For quartile 4, the range was $86.68-100 \%$ for cohort 1 and $86.68-100 \%$ for cohort 2 . In general, the two cohorts of students had similar percentages when broken into quartiles, with cohort 2 having a slightly higher range for each quartile, except for the fourth quartile.

Changes in the quartiles between the cariology case study (first assessment) in the first year and the OSCE (final assessment) in the fourth year are shown in Table 3 and Figure 1. For students in the lowest quartile (Q1) after the first assessment, 37\% (cohort 1) and $21 \%$ (cohort 2) had moved into quartile $4(\mathrm{Q} 4)$ by the final assessment. Furthermore, 35\% (cohort 1) and $27 \%$ (cohort 2 ) of students who started in quartile 2 (Q2) finished in the top quartile (Q4).

With each consecutive assessment, there was an increase of $2.9 \%(p<0.001)$ for cohort 1 (Figure 2 , panel a) and an increase of $2.2 \%(p<0.001)$ for cohort 2 (panel b). For both cohorts, the top quartile's (Q4) change in scores $(-0.15 \% ; 0.39 \%)$ did not reach statistical significance $(\mathrm{p}=0.82 ; \mathrm{p}=0.39)$ and remained the highest across the four years (Figure $3)$. However, students in the lowest quartile (Q1) increased their scores by $9.4 \%$ (cohort $1, \mathrm{p}<0.001$ ) and $4.6 \%$ (cohort $2, p=0.003$ ) with each consecutive assessment. Students in the second quartile (Q2) also increased their scores over time, but with lower gains: by $2.9 \%$ (cohort $1, \mathrm{p}<0.001$ ) and $2.4 \%$ (cohort $2, \mathrm{p}<0.001)$. For students in the third quartile (Q3), cohort 2 had a statistically significant improvement of $1.3 \%(\mathrm{p}=0.047)$, while cohort 1 had a similar trend $(0.87 \%)$ but it did not reach statistical significance $(\mathrm{p}=0.421)$.

Table 2. Mean student outcomes (percentage correct and standard deviation) on five critical thinking assessments used in study, for the two student cohorts

\begin{tabular}{|c|c|c|c|c|}
\hline \multirow[b]{2}{*}{ Assessment } & \multicolumn{2}{|c|}{ Cohort 1} & \multicolumn{2}{|c|}{ Cohort 2} \\
\hline & Mean & SD & Mean & SD \\
\hline Cariology I case study & $74.0 \%$ & $22.2 \%$ & $79.6 \%$ & $13.6 \%$ \\
\hline Cariology I final exam & $89.7 \%$ & $7.0 \%$ & $86.3 \%$ & $10.9 \%$ \\
\hline $\begin{array}{l}\text { Treatment planning } \\
\text { course final exam }\end{array}$ & $86.0 \%$ & $14.9 \%$ & $84.6 \%$ & $17.5 \%$ \\
\hline $\begin{array}{l}\text { Geriatric dentistry course } \\
\text { OSCAR assignment }\end{array}$ & $85.3 \%$ & $25.0 \%$ & $87.6 \%$ & $20.0 \%$ \\
\hline OSCE & $90.8 \%$ & $4.1 \%$ & $90.0 \%$ & $6.2 \%$ \\
\hline
\end{tabular}


Table 3. Shift of number (\%) of students in each quartile from assessment 1 (shown in assessment 1 columns) to their corresponding quartile in assessment 5 (shown in rows)

\begin{tabular}{|c|c|c|c|}
\hline \multicolumn{2}{|r|}{ Cohort 1} & \multicolumn{2}{|r|}{ Cohort 2} \\
\hline $\begin{array}{l}\text { Assessment } 5 \\
\text { Quartile } 1 \\
\text { Quartile } 2 \\
\text { Quartile } 3 \\
\text { Quartile } 4\end{array}$ & $\begin{aligned} \text { Assessment } & 1 \text { Quartile } 1 \quad(\mathrm{n}=19) \\
& 5(26 \%) \\
& 5(26 \%) \\
& 2(11 \%) \\
& 7(37 \%)\end{aligned}$ & $\begin{array}{l}\text { Assessment } 5 \\
\text { Quartile } 1 \\
\text { Quartile } 2 \\
\text { Quartile } 3 \\
\text { Quartile } 4\end{array}$ & $\begin{array}{c}\text { Assessment } 1 \text { Quartile } 1 \quad(\mathrm{n}=24) \\
12(50 \%) \\
3(13 \%) \\
4(17 \%) \\
5(21 \%)\end{array}$ \\
\hline $\begin{array}{l}\text { Assessment } 5 \\
\text { Quartile } 1 \\
\text { Quartile } 2 \\
\text { Quartile } 3 \\
\text { Quartile } 4\end{array}$ & $\begin{aligned} \text { Assessment } & 1 \text { Quartile } 2(\mathrm{n}=20) \\
& 3(15 \%) \\
& 8(40 \%) \\
& 2(10 \%) \\
& 7(35 \%)\end{aligned}$ & $\begin{array}{l}\text { Assessment } 5 \\
\text { Quartile } 1 \\
\text { Quartile } 2 \\
\text { Quartile } 3 \\
\text { Quartile } 4\end{array}$ & $\begin{array}{c}\text { Assessment } 1 \text { Quartile } 2(\mathrm{n}=11) \\
4(36 \%) \\
0(0 \%) \\
4(36 \%) \\
3(27 \%)\end{array}$ \\
\hline $\begin{array}{l}\text { Assessment } 5 \\
\text { Quartile } 1 \\
\text { Quartile } 2 \\
\text { Quartile } 3 \\
\text { Quartile } 4\end{array}$ & $\begin{array}{c}\text { Assessment } 1 \text { Quartile } 3(\mathrm{n}=31) \\
5(16 \%) \\
10(32 \%) \\
10(32 \%) \\
6(19 \%)\end{array}$ & $\begin{array}{l}\text { Assessment } 5 \\
\text { Quartile } 1 \\
\text { Quartile } 2 \\
\text { Quartile } 3 \\
\text { Quartile } 4\end{array}$ & $\begin{array}{c}\text { Assessment } 1 \text { Quartile } 3(\mathrm{n}=37) \\
9(24 \%) \\
10(27 \%) \\
8(22 \%) \\
10(27 \%)\end{array}$ \\
\hline $\begin{array}{l}\text { Assessment } 5 \\
\text { Quartile } 1 \\
\text { Quartile } 2 \\
\text { Quartile } 3 \\
\text { Quartile } 4\end{array}$ & $\begin{array}{c}\text { Assessment } 1 \text { Quartile } 4(\mathrm{n}=31) \\
6(19 \%) \\
8(26 \%) \\
6(19 \%) \\
11(35 \%)\end{array}$ & $\begin{array}{l}\text { Assessment } 5 \\
\text { Quartile } 1 \\
\text { Quartile } 2 \\
\text { Quartile } 3 \\
\text { Quartile } 4\end{array}$ & $\begin{array}{c}\text { Assessment } 1 \text { Quartile } 4(\mathrm{n}=35) \\
7(20 \%) \\
8(23 \%) \\
10(29 \%) \\
10(29 \%)\end{array}$ \\
\hline
\end{tabular}

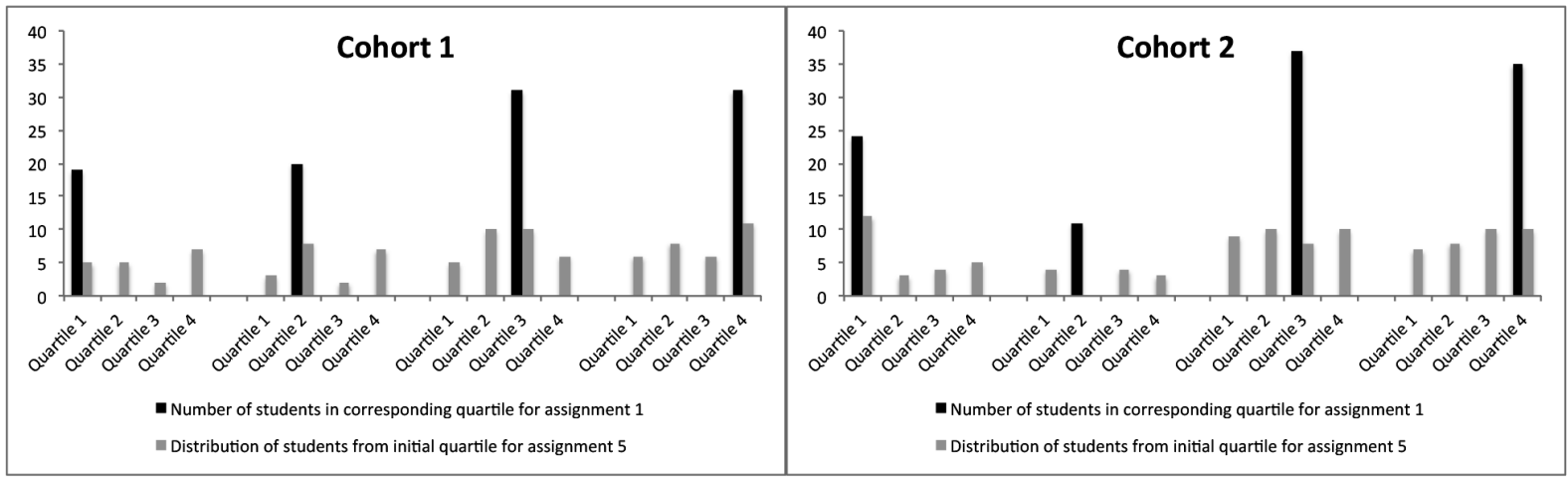

Figure 1. Shift of number of students in each quartile from assessment 1 to assessment 5 for cohort 1 (left panel) and cohort 2 (right panel)

Note: "Quartiles" ranged from 1=lowest performing students to 4=highest performing students on the baseline assessment.

\section{Discussion}

Johnsen et al. suggested that there is a need to longitudinally assess critical thinking skills across the dental curriculum in order to best measure longterm learning gains. ${ }^{5}$ In our study, for both cohorts, the students who had the lowest scores on the initial assessment of critical thinking skills showed statistically significant improvements over time. In contrast, the group of students who performed the highest on the Cariology I case study (baseline assessment) had a trend of remaining at the top for each assessment, suggesting top students with critical thinking capacities can be identified early on. Although the top quartile's change in scores did not reach statistical significance, it may be that the lowest quartiles were reaching the same level as the top quartiles 

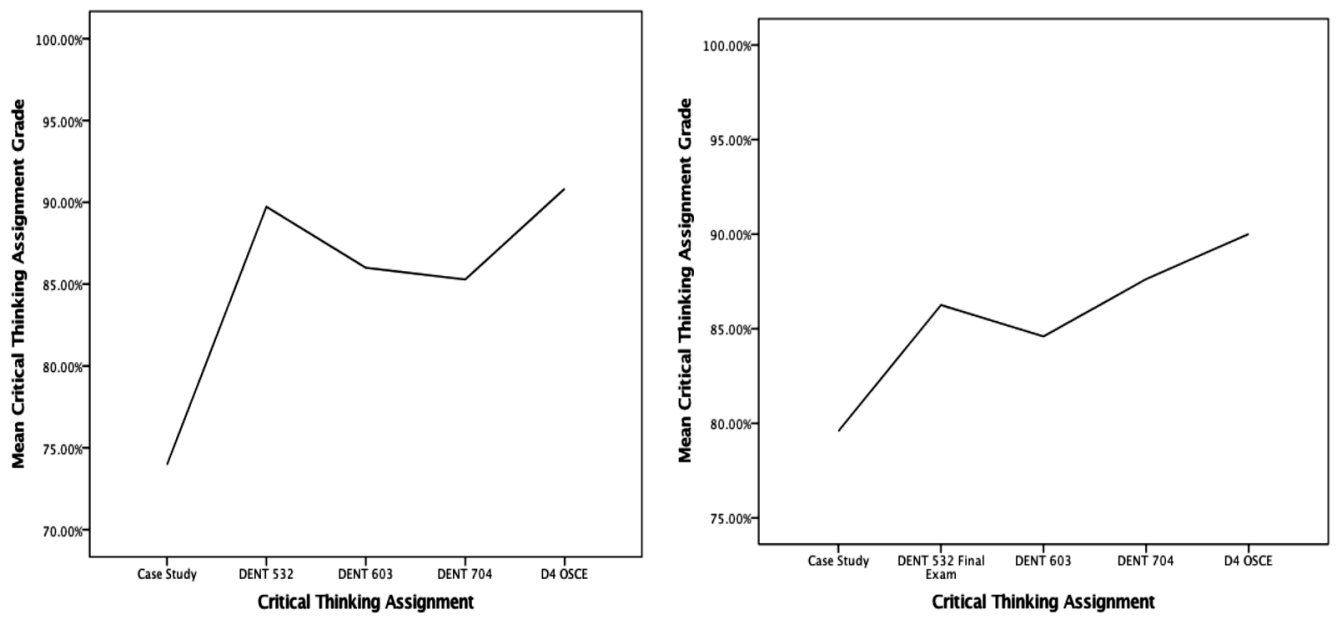

Figure 2. Change in mean grades from first to fifth assessments for cohort 1 (left panel) and cohort 2 (right panel)
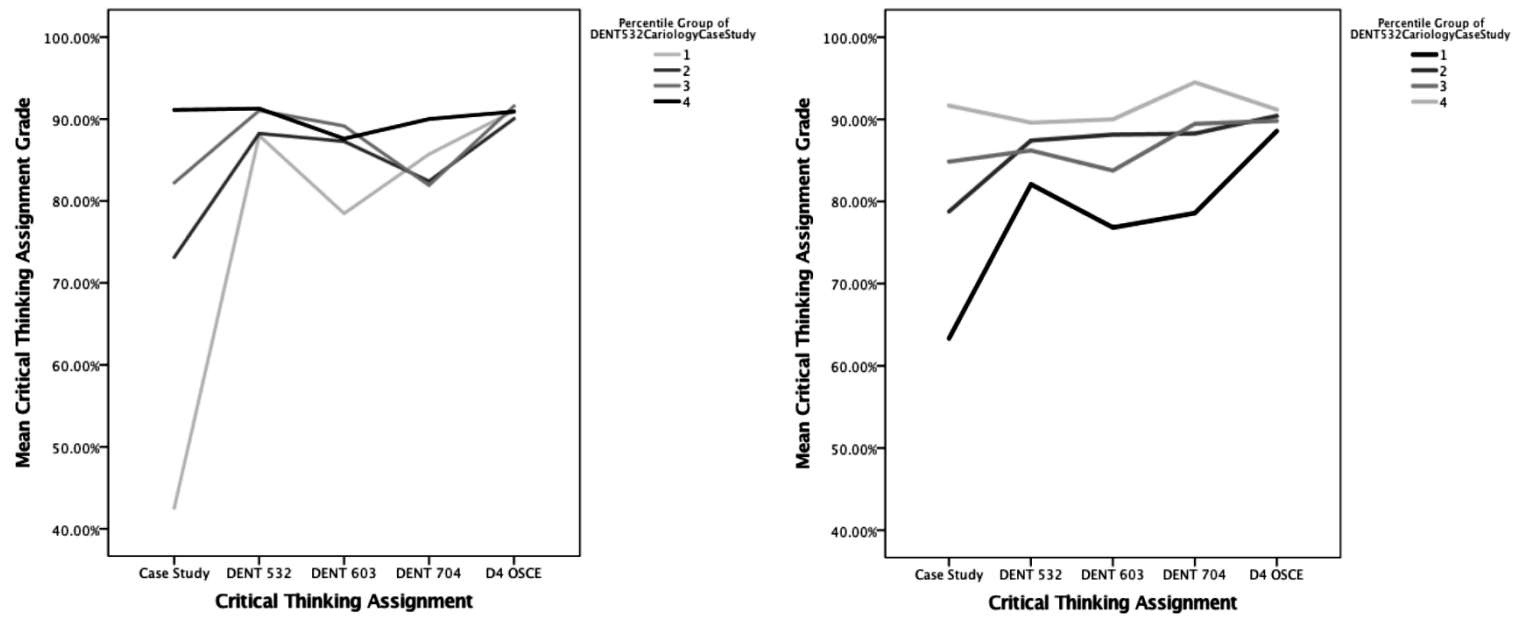

Figure 3. Change in mean grades from first to fifth assessments by quartiles based on Cariology I case study, for cohort 1 (left panel) and cohort 2 (right panel)

Note: "Percentile groups" ranged from 1=lowest performing students to $4=$ highest performing students on the baseline assessment.

prior to graduation. These results complement a preliminary study by Chambers that found students' overall performance in assessed critical thinking activities was significantly associated with their clinical competency ratings for clinical judgment and patient management in the clinical courses. ${ }^{3}$ Our analysis indicates that critical thinking improvements can also be seen longitudinally over the full course of the curriculum.

Johnsen et al. identified a current challenge in dental education as the lack of a well-defined model for assessing critical thinking and problemsolving. ${ }^{13}$ Such a model should facilitate assessment of the growth of students' critical thinking abilities over the full spectrum of the curriculum, across disciplines, and ideally with embedded and direct assessments. ${ }^{14-17}$ A model proposed by Johnsen et al. encourages validity, reliability, and comparability of critical thinking assessments across disciplines and curriculum years. ${ }^{6}$ Their model includes establishing an educational outcome, developing a learning construct, having the student apply the steps, and 
then assessing the student's performance level. In each of the steps, there can be a measure that is both objective and subjective: identification of the step (objective) and applying the step to a patient case (subjective). ${ }^{5}$ Repetition of this model enables faculty to assess a student's critical thinking performance. Implementing a construct across disciplines and years that controls for cognitive load can help the student transition from a beginner level to being proficient prior to graduation. ${ }^{5,14,15}$

The subjectivity of critical thinking and the absence of proper measurements make assessing students' competence in critical thinking difficult. ${ }^{13}$ In our study, grading rubrics were used to assess critical thinking in each course, but there were variations among them. This variation in assessment method is a limitation of our study. A related limitation was the differences in the grading faculty members and type of assessment, meaning the average scores for each assessment varied. For the most part, a single faculty member scored each of the assessments at the time they were completed in that course. However, as the assessments were chosen for our study after the students had graduated, there was no calibration of evaluators or validation of the assessments prior to scoring other than for assessment of course-specific content. Furthermore, there were small differences in the questions used for the two cohorts (e.g., although they asked the student to demonstrate the same skills and knowledge, the cases used varied). This variation may explain some of the differences seen over time and between cohorts. This inconsistency further emphasizes the need for a common rubric to assess students' critical thinking ability throughout the dental curriculum.

Despite these limitations, it is important to note that we were able to identify multiple assessments measuring critical thinking across disciplines and curriculum years. A necessary factor for the development of critical thinking identified by Fontana et al. as part of the "Advancing Dental Education in the $21^{\text {st }}$ Century" project "is a culture of inquiry with a substantive, engaged faculty in a respectful environment." 18 While most schools are making advances in a culture of inquiry and looking beyond courses and disciplines, the dental school culture remains strongly organized around courses and disciplines, while critical thinking crosses disciplines. Our study did not evaluate the culture of the academic environment. However, the improvements in critical thinking for the students and the presence of critical thinking assessments across disciplines across four years sup- port the notion that a culture of inquiry, collaboration, and communication about critical thinking exists to at least some degree in the current system. Whether this current culture was the cause for the improvement is unknown.

The results of our study indicate that critical thinking assessments implemented early in the curriculum can identify students who may require additional assistance prior to reaching the clinics. The study also demonstrates the potential for improvement of this group's critical thinking skills, as long as the curriculum and assessments are carefully designed to give students the opportunity to practice these skills. For those students who demonstrate sufficient critical thinking skills early on, it can be expected that they will continue to do well again, if the curriculum is designed to reinforce those competencies. Engaging students in multiple learning situations that require critical thinking will enable them to adapt to clinical scenarios in the future. Thus, not relying on a single assessment improves the likelihood of gaining meaningful insight in an area in which a single assessment is inherently incomplete in gaining insight into overall critical thinking abilities.

\section{Conclusion}

This study's results showed that the students who had difficulty with critical thinking early in the curriculum improved in their critical thinking skills as they progressed. Future research could be directed to development of a standardized rubric, so that critical thinking can be assessed in a more comparable manner across courses. Such a rubric would also help educators to monitor students' abilities to problemsolve and think critically as they progress through the dental curriculum.

\section{Acknowledgments}

We would like to acknowledge the University of Michigan Office of the Provost, the Center for Research on Learning and Teaching, and the Provost's Learning Analytics Task Force for funding this project through the 2013-14 Investigating Student Learning (ISL) Program.

\section{REFERENCES}

1. Hart Research Associates. It takes more than a major: employer priorities for college learning and student success. Washington, DC: Association of American Colleges and Universities and Hart Research Associates, 2013. 
2. Commission on Dental Accreditation. Accreditation standards for dental education programs. Chicago: American Dental Association, 2016.

3. Chambers DW. Lessons from students in a critical thinking course: a case for the third pedagogy. J Dent Educ 2009;73(1):65-82.

4. American Dental Education Association Commission on Change and Innovation in Dental Education. Overview of critical thinking skills. At: www.adea.org/adeacci/ Resources/Critical-Thinking-Skills-Toolkit/Pages/Overview-of-Critical-Thinking-Skills.aspx. Accessed 28 Apr. 2016.

5. Johnsen DC, Finkelstein MW, Marshall TA, Chalkley YM. A model for critical thinking measurmement of dental student performance. J Dent Educ 2009;73(2):177-83.

6. Johnsen DC, Lipp MJ, Finkelstein MW, Cunningham MA. Guiding dental student learning and assessing performance in critical thinking with analysis of emerging strategies. J Dent Educ 2012;76(2):1548-58.

7. Duron R, Limbach B, Waugh W. Critical thinking framework for any discipline. IJTLHE 2006;17(2):160-6.

8. Elder L, Paul R. Critical thinking development: a stage theory. 2010. At: www.criticalthinking.org/pages/criticalthinking-development-a-stage-theory/483. Accessed 11 June 2013.

9. Bloom BS. Taxonomy of educational objectives: the classification of educational goals. Handbook 1, cognitive domain. New York: David McKay Co., 1956.
10. Fontana M, Guzman-Armstrong S, Schenkel AB, et al. Development of a core curriculum framework in cariology for U.S. dental schools. J Dent Educ 2016;80(6):705-20.

11. Zartman RR, McWhorter AG, Seale S, Boone WJ. Using OSCE-based evaluation: curricular impact over time. J Dent Educ 2002;66(12):1323-30.

12. McComas MJ, Wright RA, Mann NK, et al. M-OSCE as a method to measure dental hygiene students' critical thinking: a pilot study. J Dent Educ 2013;77(4):485-93.

13. Johnsen DC, Williams JN, Baughman PG, et al. New dental accreditation standard on critical thinking: a call for learning models, outcomes, assessments. J Dent Educ 2015;79(10):1137-9.

14. Johnsen DC. Critical thinking: focal point for a culture of inquiry. In: Boyle C, ed. Student learning: improving practice. Hauppauge, NY: Nova Science Publishers, 2013:151-70.

15. Postma TC, White JG. Developing clinical reasoning in the classroom: analysis of the 4C/ID-model. Eur J Dent Educ 2015;19(2):74-80.

16. Maki PL. Assessing for learning: building a sustainable commitment across the institution. Sterling, VA: Stylus, 2004.

17. Gonzalez-Cabezas C, Anderson OS, Wright MC, Fontana M. Exam questions developed by students lead to higher cognitive level of learning. J Dent Educ 2015;79(11): 1295-304

18. Fontana M, Gonzalez-Cabezas C, de Peralta T, Johnsen D. Dental education required for the changing dental environment. J Dent Educ 2017;88(8 Suppl):eS153-61. 